\title{
Ein Beitrag zur Morphogenese der Pars tuberalis von Hypophysis cerebri bei den verschie= denen japanischen Amphibien.
}

\author{
Von \\ A. o. Prof. Dr. R. Takasima und Dr. S. Yuba. \\ (Aus dem II. Anatomischen Institut der kaiserlichen Universität zu Osaka \\ (Prof. Dr. T. Tomita)).
}

Inhalt.

I. Einleitung. . . . . . . . . . . . . . . . . . . . 665

II. Betrachtungen . . . . . . . . . . . . . . . . . . . . 667

III. Zusammenfassung . . . . . . . . . . . . . . . . . . 672

Literaturverzeichnis . . . . . . . . . . . . . 673

\section{Einleitung.}

Anschliessend an die Forschungen Atwells (1918, 1921), der zuerst bei R. pipiens und Amblystoma punctatum sowohl auf das Vorhandensein als auch die Morpho- $\mathrm{u}$. Histogenese der Pars tuberalis der Hypophyse aufmerksam machte, haben einerseits Sumi (1924), Prof. Kuto (1934) und Sato (1934) bei verschiedenen Urodelen, (Onychodactylus japonicus, Megalobatrachus japonicus, Hynobius nebulosus, Diemyctylus pyrrhogaster u. a.) und anderseits Sato $(1934,1935)$ und unsere Kollegen Terato, Yamada, Imamura und Yuba im letzten Jahre bei einigen anuren Amphibien (B. vulgaris japonicus, Rhacophorus schlegelii arborea und R. nigromaculata), den genannten Abschnitt der Hypophyse entwicklungsgeschichtlich genau studiert. Sämtliche oben genannte Autoren haben übereinstimmend berichtet, dass die Pars tuberalis sekundär aus der Vorderlappenanlage gebildet wird, wenn auch die Meinungen über den Zeitpunkt, wo sie zuerst in Erscheinung tritt, verschieden sind. 
Nach Atwells Angaben tritt die Pars tuberalis bei Rana pipiens ziemlich früh, und zwar bei $7 \mathrm{~mm}$ langer Kaulquappe und bei Amblystoma punctatum erst später (bei einer Länge von $14-15 \mathrm{~cm}$ ) auf, während sie bei von Sumi, Kuto und Sato zur Verfügung gehabten japanischen Amphibien stets erst im Stadium der Metamorphose, bei allen von uns beobachteten dagegen ausserordentlich früh in Erscheinung tritt.

Was zunächst die Entstehungsweise derselben anbetrifft, so stimmen alle früheren Angaben, ausser den von Atwell gemachten, vollkommen überein, dass die Pars tuberalis sich unmittelbar aus dem kranialen Ende der Vorderlappenanlage in Gestalt zweier hornartiger Fortsäıe bilden soll. Demgegenüber haben unsere Kollegen, wie sie bereits mitgeteilt haben, beobachtet, dass die linke und rechte Pars tuberalis, wenigstens bei von ihnen untersuchten drei verschiedenen Anurenarten, immer sekundär aus der von der Vorderlappenanlage herausgebildeten gemeinschaftlichen Anlage in Erscheinung treten, wenn auch die Entstehungsweise sich je nach der Tierart ziemlich stark unterscheidet.

Es wird jedoch im allgemeinen für ziemlich bestimmt angenommen, dass die Pars tuberalis und der Vorderlappen, bei Urodelen ausschliesslich des Riesensalamanders, lebenslang und bei Anuren nur im larvalen Stadium, miteinander verbunden sind, wenn auch Sumi kürzlich eine bemerkenswerte Annahme, die wir leider, wie später von uns erwähnt, nicht bestätigen konnten, veröffentlicht hat. Er unterscheidet dabei auf Grund Atwells (1918, 1921) und seiner eigenen Ergebnisse an der Hypophyse der Amphibien drei Typen, u. zw. zwei Typen und eine Unterart bei Urodelen und ein Typus bei Anuren. Beim ersten ,U-Typus“ der Urodelen ist die Pars tuberalis wie bei Amblystoma, Necturus und Amphiuma, stets mit dem Vorderlappen U-förmig und bei einer Unterart (I-Typus) des L-Typus, wie \%. B. heim Hynobius und Onychodactylus V-förmig verbunden, während sie beim zweiten ,, O-Typus“ der Urodelen ganz (beim Riesensalamander) oder teilweise (beim Triton) fehlt und beim ,Ö-Typus“ der Anuren stets vom Torderlappen getrennt anzutreffen ist. Dabei ist die Richtigkeit seiner Angabe, dass er beim Diemyctylus zuweilen eine Übergangsform zwischen dem U-Typus der Urodelen und dem Ö-Typus der Anuren unterscheidet, wenn er auch die Entstehungsweise der Pars tuberalis nicht berücksichtigt hat, nicht erwiesen. Bei dieser Übergangsform soll die Pars tuberalis, wenigstens nach Sumis Ergebnissen bald beiderseitig, bald nur einseitig vorhanden sein.

In Anbetracht dieser Feststellungen ist es zweckmässig und nicht uninteressant, die Entstehungsweise der Pars tuberalis und ihren weiteren Entwicklungsgang bei verschiedenen, vor allem bei bisher noch nicht 
untersuchten Amphibien, den schon bereits bei anderen Amphibien von verschiedenen Autoren erzielten Resultaten zu vergleichen, um die Arteigenschaft und deu phylogenetischen Zusammenhaing zwischen denselben bei verschiedenen urodelen und anuren Amphibien festzustellen und die morphogenetischen Einzelheiten klarzustellen.

\section{Betrachtungen.}

Wir haben hier nicht nur drei einheimische Anuren (Bufo vulgaris japonicus, Rana nigromaculata und Rhacophorus schlegelii arborea) und eine koreanische Anurenart (Cacopoides tornieri), die Takasima, einer der Autoren, in der letzten Mitteilung aufgeführt hat, sondern auch eine koreanische Urodelenart (Hynobius leechii), welche vor einigen Jahren vom anatomischen Institut zu Keijo (in Korea) verschafft wurde, zur Verfügung gehabt.

Die Pars tuberalis der von Atwell, Sumi, Kuto und Sato bereits untersuchten anuren und urodelen Arten wird stets ziemlich später, und zwar nahe am Ende der Metamorphose der Larven von der Vorderlap-

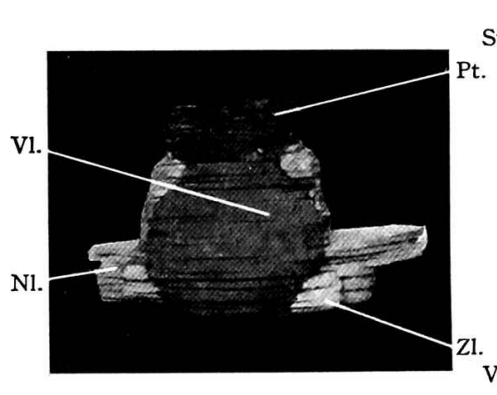

Fig. 1.

Rhacophorus schlegelii arborea. (Kaulquappe von $6,0 \mathrm{~mm}$ (SchnauzenAnuslänge)-16,0 mm (SchnauzenSchwanzlänge)).

Wachsplattenmodell der gesamten Hypophyse.

(Ventrale Ansicht).

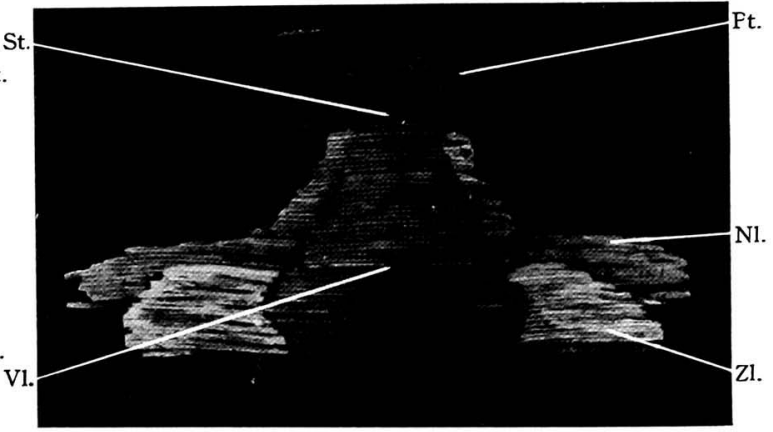

Fig. 2.

Rhacophorus schlegelii arborea. (Kaulquappe von 12,0-28,0 $\mathrm{mm}$ Länge).

Wachsplattenmodell der gesamten $\mathrm{H}_{y}$ pophyse. (Ventrale Ansicht).

Pt. = Pars tuberalis,

Vl. = Vorderlappen,

$Z \mathrm{l}$. = Zwischenlappen,

Nl. = Nervenlappen,

St. $=$ Stiel der Pars tuberalis

penanlage sekundär gebildet, während sie sich bei von uns untersuchten Anurenarten, wie bei Rhacophorus, Rana, Bufo und Cacopoides immer frühzeitig und zwar schon am Ende des I. Stadiums (ca. 5-10 mm, 
Rumpflänge) aus dem kranialen Ende der Vorderlappenanlage zu entwickeln beginnt (Fig. 1, 4, 7, 10), um später gegen Ende der Metaınorphose vom Vorderlappen abgeschnürt zu werden (Fig. 3, 6, 9, 12).

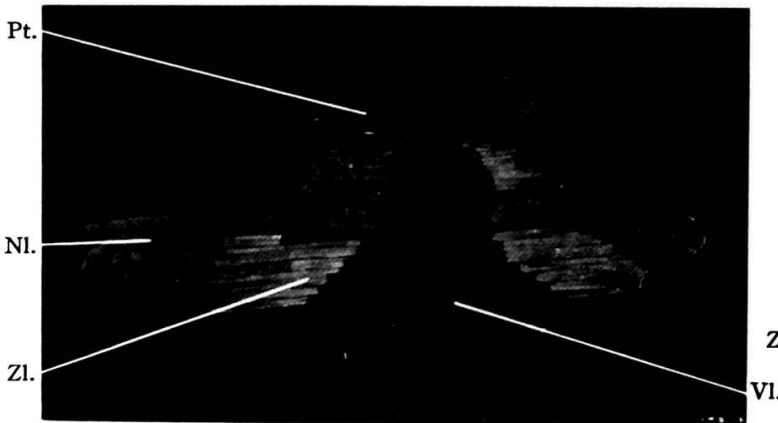

Fig. 3.

Rhacophorus schlegelii arborea. (Kaulquappe von 15,0-30,0 mm Iänge).

Wachsplattenmodell der gesamten Hypophyse. (Ventrale Ansicht).

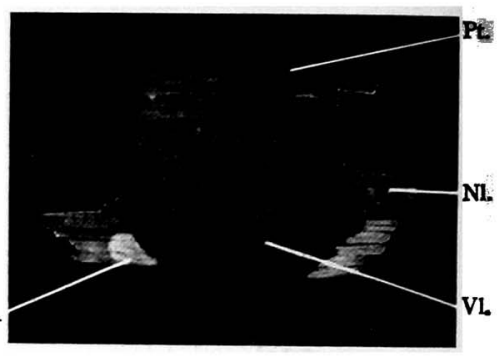

Fig. 4.

Rana nigromaculata. (Kaulquappe von $9,0-21,0 \mathrm{~mm}$ Iänge).

Wachsplattenmodell der Hypophyse. (ventral gesehen).

Fs steht immer fest, dass die erste Anlage der Pars tuberalis bei Beginn ihrer Differenzierung bei allen Spezies der Anuren, wie bei Rhacophorus, Bufo und Rana, die koreanischen Cacopoides ausgenommen, als ein ein-

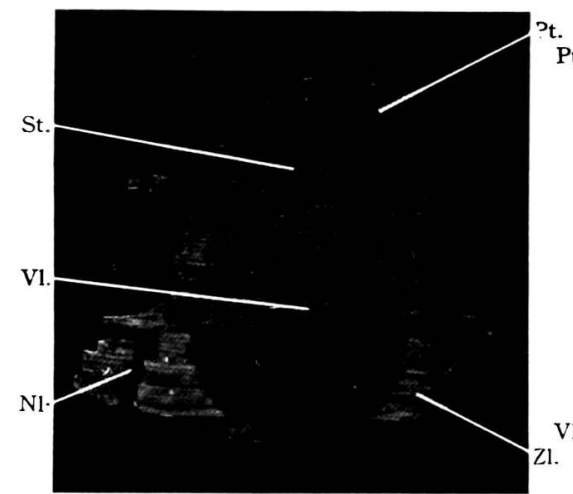

Fig. :).

Rana nigromaculata.

(Kaulquappe ron 16,0-36,0 mm Jänge). Wachsplattenmodell der Hypophyse. (ventral geschen).

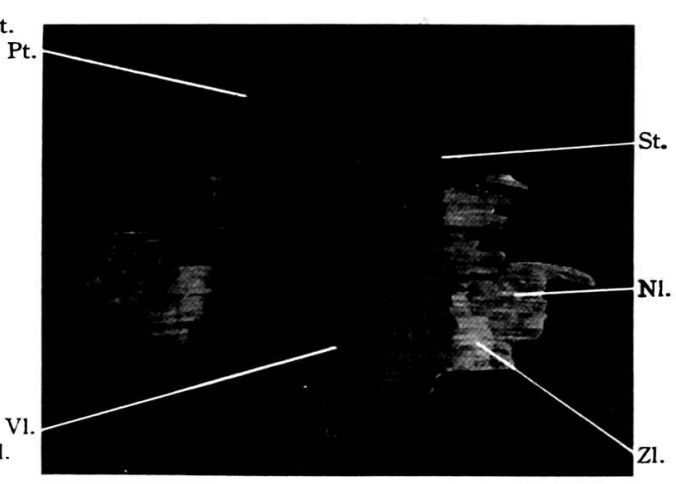

Fig. 6.

Rana nigromaculata.

(Kaulquappe von 17,0-41,0 mm I änge). Wachsplattenmodell der Hypophyse. (ventral gesehen).

heitliches Gebilde auftritt, un später mit ihrer weiteren Entwicklung in linke und rechte Hälften gespalten und schliesslich vom Vorderlappen abgetrennt zu werden. Wir haben dabei noch eine merkwürdige Er- 
Ein Beitrag zur Morphogenese der Pars tuberalis von Hypophysis cerebri, etc. 669

scheinung beobachtet, nämlich dass die Pars tuberalis in ihrem genetischen Detail je nach den Tierspezies ausserordentlich verschieden ist.

Die erste, allen Spezies eigene Anlage der Pars tuberalis weist beim

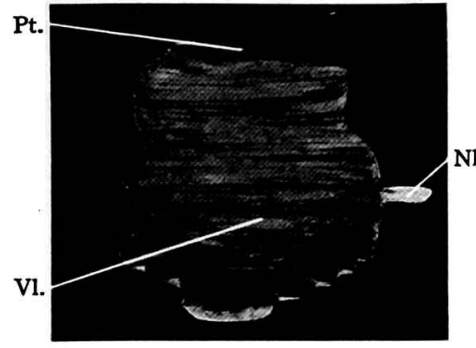

Fig. 7.

Bufo vulgaris japonicus. (Kaulquappe von 7,0-18,0 $\mathrm{mm}$ Iänge). Wachsplatten modell von Hypophyse. (Ventralansicht).

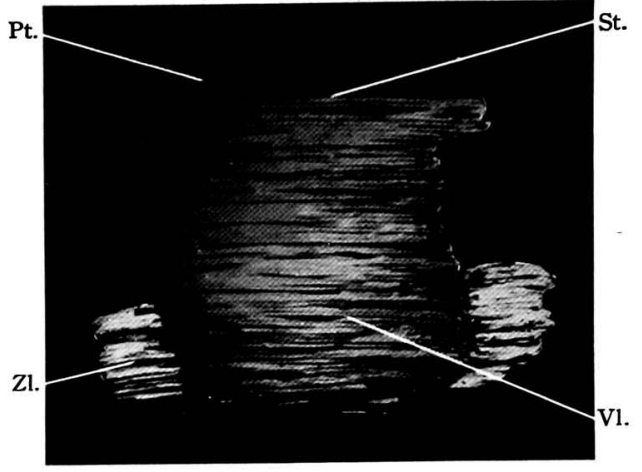

Fig. 8.

Bufo vulgaris japonicus. (Kaulquappe von 11,0-27,0 min Iänge). Wachsplattenmodell von Hypophyse. (Ventralansicht).

Rhacophorus in der Regel Scheibenform auf und ist nicht selten durch einen schmalen und kurzen Stiel mit der Vorderlappenanlage verbunden (Fig. 1), weil die erste Anlage von der Vorderlappenanlage etwas früher

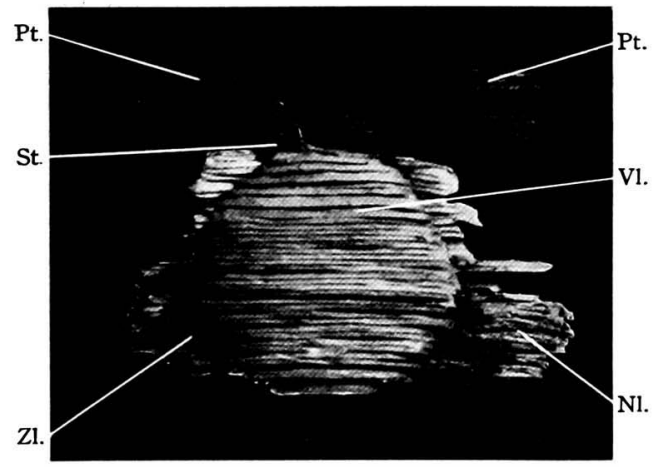

Fig. 9.

Bufo vulgaris japonicus. (Kaulquappe von 10,0-10,0 $\mathrm{mm}$ I.änge). Wachsplattenmodell von Hypophyse. (Ventralansicht).

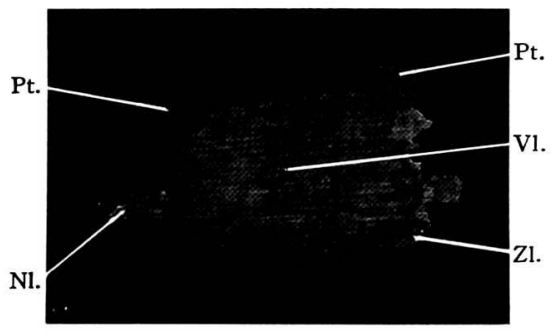

Fig. 10.

Cacopoides tormieri. (Kaulquappe von 10,0-21,0 mm Länge). Wachsplattenmodell ron Hypophyse. (Ventrale Ansicht).

abgeschnürt wird, als sie in die linke und rechte Hälfte zerteilt wird. Der Stiel, der beim Rhacophorus eigentümlich gut entwickelt ist, ist beim Bufo so schwach entwickelt, dass er nicht selten übersehen wird (Fig. 7). 
Er tritt nur vorübergehend am Anfang der Entwicklung der Pars tuberalis als. ein schmales bandartiges Gebilde, welches längs dem vordersten Rande der Vorderlappenanlage liegt, auf, um zugleich die gemeinsame Anlage der beiderseitigen Partes tuberales aufzuweisen und den letzteren aus dem

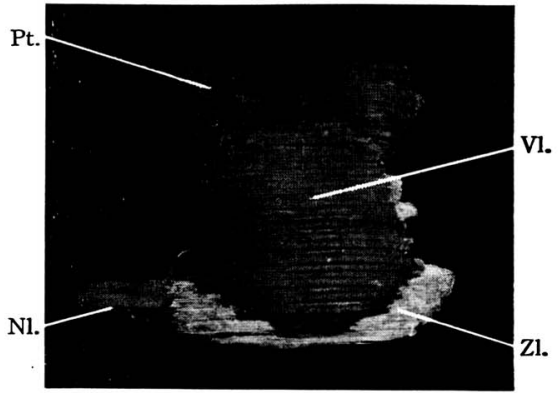

Fig. 11.

Cacopoides tornieri.

(Kaulquappe von 12,0-28,0 $\mathrm{mm}$ Iänge). Wachsplattenmodell von Hypophyse. (Ventrale Ansicht).

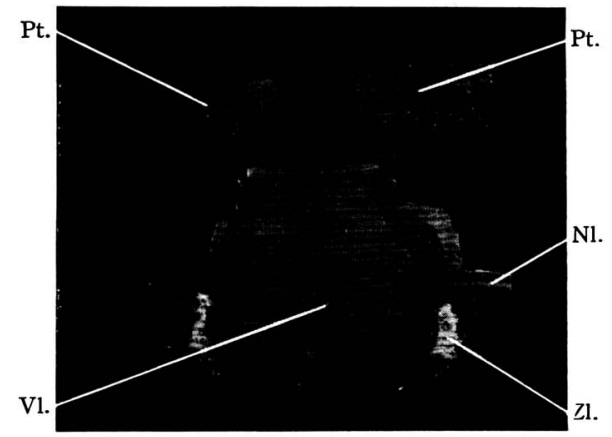

Fig. 12.

Cacopoides tornjeri.

(Kaulquappe von 19,0-20,0 mm Iänge). Wachsplattenmodell von Hypophyse. (Ventrale Ansicht).

linken und rechten Ende der ersten Anlage hornartig herausdifferenzieren zu lassen (Fig. 8). Demgegenüber bildet die gemeinsame Anlage der Rana eine $Z$ wischenform der eben erwähnten zwei Arten, indem sie eine

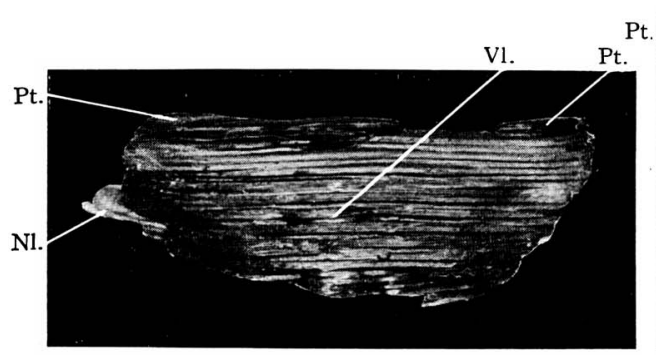

Fig. 13.

Hynobius leechii. (Larve von 8,5-12,0 $\mathrm{mm}$ Länge). Wachsplattenmodell der Hypophyse. (Ventralansicht).

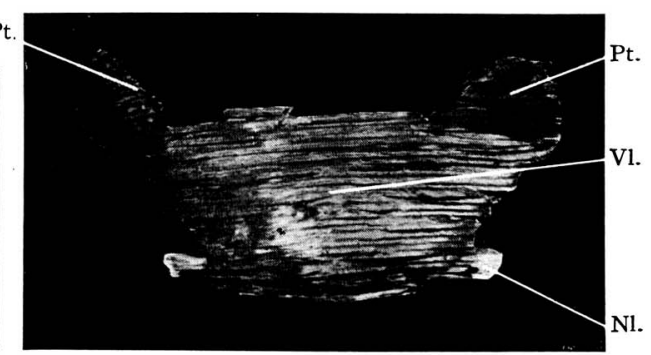

Fig. 14.

Hynobius leechii. (Iarve von 11,0-:20,0 $\mathrm{mm}$ Iänge). Wachsplattenmodell der Hypophyse. (Ventralansicht).

dünne quadratische Platte aufweist und länger als beim Rhacophorus mit der Vorderlappenanlage zusammenhängt (Fig. 4). Sie wird also, wie aus beistehenden Figuren ersichtlich ist, zuerst in zwei Hälften zerteilt und dann von der Vorderlappenanlage abgetrennt (Fig. 5-6). 
Die obigen Befunde lassen es höchstwahrscheinlich erscheinen, dass die Pars tuberalis bei den oben beschriebenen drei Anurenarten (Rhacophorus, Rana und Bufo) sich erst sekundär aus der gemeinsamen Anlage

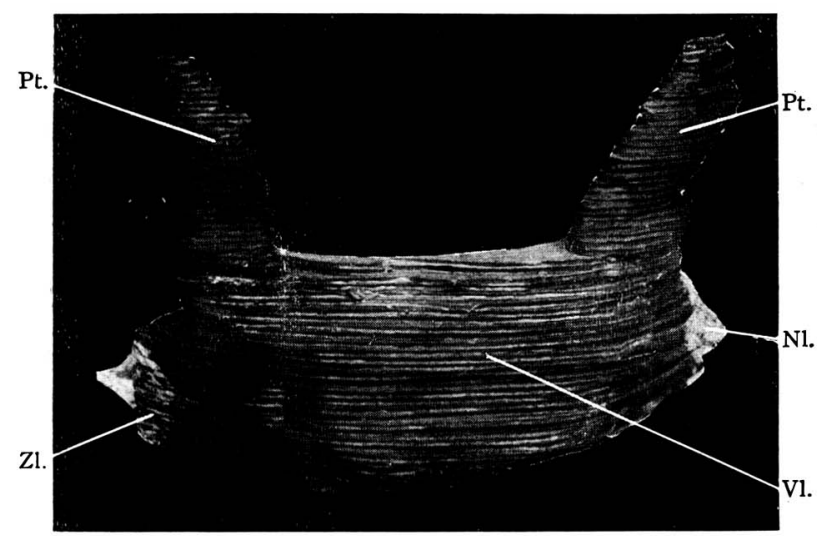

Fig. 15.

Hynobius leechii.

(Jarve von $23,0-46,0 \mathrm{~mm}$ Iänge).

Wachsplattenmodell der Hypophyse.

(Ventralansicht).

herausbildet, wemn auch die Art ihrer Entstehung je nach der Tierart ziemlich stark verschieden ist.

Wenn man jetzt die Pars tuberalis der Cacopoides genauer betrachtet, so sieht man wider Erwarten, dass die Art ihrer Entstehung im Vergleich zu der der oben erwähnten drei Arten Anuren gänzlich verschieden ist. Sie entwickelt sich bei dieser Tierart unmittelbar aus dem kranialen Teil der Vorderlappenanlage als ein paariges hornartiges Gebilde, ohne dabei eine gemeinsame Anlage, die den oben erwähnten drei Anurenarten eigentümlich ist, aufzuweisen (Fig. 10-11). Diese Eigenart ist mit der der schon bekamnten Urodelen und unseres koreanischen Hynobius leechii ganz identisch (Fig. 13), wenn sie auch später von diesen ziemlich stark abweicht. Die Pars tuberalis der Cacopoides wird im Laufe ihrer weiteren Entwicklung allmählich an Grösse zunehmen, um schliesslich bei Beginn der Metamorphose der Kaulquappe vollständig vom Vorderlappen abgeschnürt zu werden (Fig. 12), während sie bei unserem Hynobius, wie aus Fig. 13-15 ersichtlich, stets ebenso wie beim ,U-Typus“ der oben erwähnten anderen Urodelen mit dem Vorderlappen irnmer zusammenhängend bleibt. Sie wird sich jedoch beim Hynobius leechii wesentlich schneller und stärker als bei den Anuren entfalten (Fig. 13-14-15), um 
sich dabei vom Vorderlappen hornartig auszustrecken und U-förmig mit dem letzteren zusammenhängend zu bleiben.

\section{Zusammenfassung.}

Fassen wir jetzt die im vorigen Kapitel beschriebenen einzelnen Befunde zusammen, so können wir, auf Grund unserer Untersuchungen, die von Sumi gemachten Beobachtungen bestätigend ammehmen, dass die Pars tuberalis der Amphibien in zwei Grundformen eingeteilt wird, und zwar, dass die der Anuren dem Ö-Typus und die der Urodelen dem U-Typus angehört.

Es ist jedoch besonders merkwürdig, dass eben bei dem Ö-Typus der Anuren, der früher bei allen Anuren für gleichartig angesehen wurde, wenigstens nach der Art der Entstehung einige Untertypen zu unterscheiden sind. Die beiden extremen Formen kann man bei Rhacophorus bezw . bei Cacopoides und die Zwischenform bei Rana und Bufo beobachten. Die Pars tuberalis der Cacopoides, welche eine der beiden extremen Formen aufweist, entwickelt sich wie schon oben beschrieben, genau so wie bei unserem koreanischen Hynobius (Fig. 10-12, Fig. 13-15) und bei verschiedenen anderen Urodelen. Sie tritt schon vom Anfang ihrer Entstehung an als zweiteiliges hornartiges Gebilde auf, welches unmittelbar und nicht wie beim Rhacophorus (Fig. 1-2) durch einen gemeinschaftlichen Stiel mit dem Vorderlappen verbunden ist, wenn sie auch bei vollendeter Entwicklung von letzterem, ähnlich wie bei anderen Anuren, vollständig abgeschnürt wird (Fig. 10-12).

Demgegenüber entwickelt sich die Pars tuberalis des Rhacophorus, welche die andere der beiden extremen Formen aufweist, erst sekundär aus einer den linken und rechten Tuberales gemeinschftlichen Anlage in eigentümlicher Weise (Fig. 1-3). Die genannte gemeinschaftliche Anlage des Rhacophorus ist insofern merkwürdig, als sie bei dieser Art im Vergleich zu der der anderen Anuren, wie Rana und Bufo (Fig. 4-6, Fig. 7-9), welche eine Übergangsform zwischen Cacopoides und Rhacophorus aufweisen, besonders stark entwickelt ist, indem sie, ehe sie in zwei Hälften zerlegt, vom Vorderlappen stark abgeschnürt und durch einen schmalen Stiel mit dem Vorderlappen verbunden wird (Fig. 1-2). Die eben erwähnte gemeinschaftliche Anlage, d.h. die erste Anlage der Pars tuberalis, wird bei beiden Zwischenformen dagegen stets zuerst in linke und rechte Hälften geteilt und später vom Vorderlappen abgetrennt, während sie sich bei Rana ungefähr ähnlich wie beim Rhacophorus und beim Bufo sowie bei den Cacopoides verhält. 
Ein Beitrag zur Morphogenese der Pars tuberalis ron Hypophysis cerebri, etc. 673

In Anbetracht dieser Tatsache ist es höchstwahrscheinlich, dass die Pars tuberalis der Cacopoides, wenigstens von unserem morphogenetischen Standpunkt aus betrachtet, eine deutliche Übergangsform zwischen den Urodelen und Anuren darstellt, wie aus den beigefügten Schernata klar

\section{Schematische Darstellung der Morphogenese der Pars tuberalis von Hypophysis} cerebri bei den verschiedenen Amphibienarten.

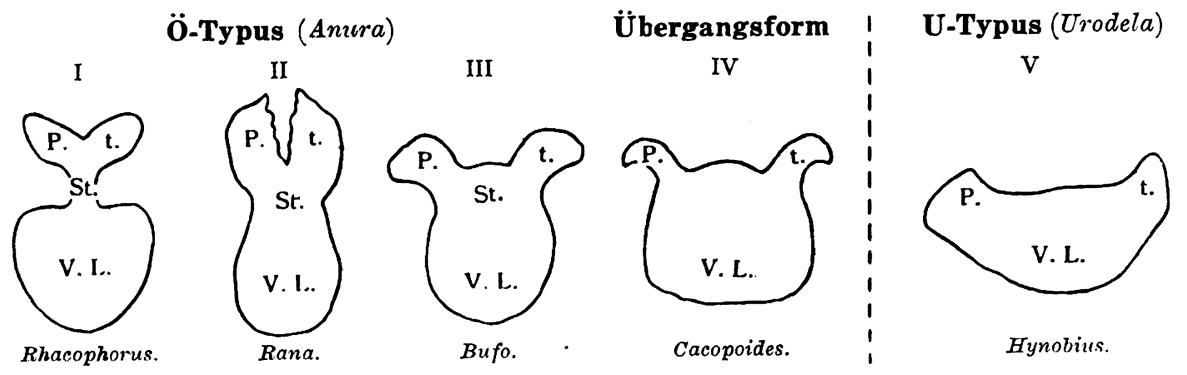

N. B. : P.t. = Pars tuberalis, St. = Stiel $\mathrm{zwischen} \mathrm{P.} \mathrm{tuberalis} \mathrm{u.} \mathrm{Vorderlappen,}$ V.L. $=$ Vorderlappen.

ersichtlich ist, trotzdem Sumi früher eine solche Zwischenform wegen 15\%-igen Fehlens der Pars tuberalis beim Diemyctylus aufsuchte. Seine Zwischenform ist, wenigstens unserer Meinung nach, nichts anderes als eine Übergangsform zwischen dem O-Typus der Riesensalamander, bei dem die Pars tuberalis gänzlich fehlt, und dem U-Typus (bei Amblystoma, Necturus und Amphiuma) oder dem Y-Typus (bei Hynobius und Onychodactylus), bei denen die Pars tuberalis stets mit dem Vorderlappen zusammenhängt, weil er nur die Pars tuberalis nach vollendeter Entwicklung untersuchte ohne dabei die genetischen Einzelheiten zu berücksichtigen. Zusammenfassend möchten wir nochmals hervorheben, dass die Pars tuberalis der Cacopoides die eigentliche Übergangsform zwischen den urodelen und anuren Amphibien darstellt, während sie im ausgebildeten Zustand dem Ö-Typus der sonstigen Anuren angehört.

\section{Literaturverzeichnis.}

1) Atwell, W. J., 1918, Anat. Rec., Vol. 15. The morphogenesis of the hypophysis in the anura.

2) Derselbe, 1921, ditto, Vol. 22. The morphogenesis of the hypophysis in the tailed amphibia.

3) Kuto, T., 1934, Arbeiten aus dem anat. Inst. d. med. Akad. zu Niigata, Bd. 4. Über die Entwicklung der lungenlosen Salamander. 
4) Sato, K., 1934, Okayama Igakkai-Zasshi, Jg. 46, Nr. 1. Studien über die Entwicklung der Hypophysenanlage. I. Mitt. : Bei den Amphibien, besonders den Embryonen von $B$. vulgaris japonicus.

5) Derselbe, 1934, ditto, Nr. 11. Studien über die Entwicklung der Hypophysenanlage: II. Mitt. : Bei den Urodelen, besonders Hynobius, welche im Regierungsbezirk Okayama leben.

6) Derselbe, 1935, ditto, Jg. 47, Nr. 1. Studien über die Morphologie und Histologie der Anurenhypophyse (III. Mitteilung).

7) Sumi, R., 1924, Folia Anat. Japon., Vol. 2. On the morphogenesis of the epithelial Hypophysis of the tailed Amphibia.

8) Derselbe, 1926, ditto, Vol. 4. Beiträge zur Morphogenese der epithelialen Hypophyse der Urodelen.

(1) Takashima, R. und Terato, K., 1935, Mitt. d. med. Ges. zu Osaka, Bd. 34, H. 4. Über den Nervenlappen der Anurenhypophyse.

10) Iieselben, 1936, Folia Anat. Japonica, Bd. XIV. Eine kurze Mitteilung über die Entwicklung des Nervenlappens von Hypophysis cerebri bei einigen japanischen Anurenarten.

11) Terato, K., 1935, Journ. of the Kumamoto Med. Society, Vol. XI, Nr. 5. Embryologische Studien über die Hypophysis cerebri bei der japanischen Kröte. I. Mitt.: Über die larvale Entwicklung der Hypophyse.

12) Derselbe, 1935, ditto. Embryologische Studien über die Hypophysis cerebri bei ler japanischen liröte. II. Mitt.: Über die postlarvale Entwicklung der Hypophyse.

13) Terato, K. und Yamada, K., 1935, Osaka Ijishinshi (Originale Auflage), Bd. 6, H. 6. Über die Fntwicklung der Hypophyse von Rhacophorus scllegelii arborea.

14) Terato, K. und Imamura, S., 1935, ditto. Über die Entwicklung der Hypophyse von Rana nigromaculata.

15) Yamada, K., 1936, ditto, Bd. 7, H. 6. Üher die Entwicklung der Hypophyse von Hynobius leechii.

16) Yuba, S., 1935, Mitt. d. med. Ges. zu Osaka, Bd. 34, H. 10. Über die Entwicklung der Hypophysis cerebri bei in Sïd-Korea spezifischen Cacopoides tornieri. 